JOURNAL OF

SYMPLECTIC GEOMETRY

Volume 12, Number 1, 105-123, 2014

\title{
THE HAMILTONIAN GEOMETRY OF THE SPACE OF UNITARY CONNECTIONS WITH SYMPLECTIC CURVATURE
}

\author{
Joel Fine
}

Let $L \rightarrow M$ be a Hermitian line bundle over a compact manifold. Write $\mathcal{S}$ for the space of all unitary connections in $L$ whose curvatures define symplectic forms on $M$ and $\mathcal{G}$ for the identity component of the group of unitary bundle isometries of $L$, which acts on $\mathcal{S}$ by pullback. The main observation of this note is that $\mathcal{S}$ carries a $\mathcal{G}$-invariant symplectic structure, there is a moment map for the $\mathcal{G}$-action and that this embeds the components of $\mathcal{S}$ as $\mathcal{G}$-coadjoint orbits. Restricting to the subgroup of $\mathcal{G}$ which covers the identity on $M$, we see that prescribing the volume form of a symplectic structure can be seen as finding a zero of a moment map. When $M$ is a Kähler manifold, this gives a moment-map interpretation of the Calabi conjecture. We also describe some directions for future research based upon the picture outlined here.

\section{Introduction}

Let $L \rightarrow M$ be a Hermitian line bundle over a compact $2 n$-dimensional manifold. We assume throughout that $c_{1}(L)$ contains symplectic forms. This note investigates the space $\mathcal{S}$ of all unitary connections $A$ in $L$ for which $\omega_{A}=$ $\frac{i}{2 \pi} F_{A}$ is a symplectic form on $M$. Write $\mathcal{G}$ for the identity component of the group of all unitary bundle isometries (not necessarily covering the identity on $M$ ). In other words, we consider those diffeomorphisms of $L$ which send fibres to fibres by unitary isomorphisms and which can be connected by a path of such diffeomorphisms to the identity. The group $\mathcal{G}$ acts on $\mathcal{S}$ by pull-back. The main observation of this note is the following.

\section{Theorem 1.}

- $\mathcal{S}$ carries a $\mathcal{G}$-invariant symplectic form;

- There is an equivariant moment-map $\mu: \mathcal{S} \rightarrow \operatorname{Lie}(\mathcal{G})^{*}$ for the $\mathcal{G}$ action; 
- The map $\mu$ embeds each component of $\mathcal{S}$ as a coadjoint orbit of $\mathcal{G}$.

- The cover $\mathcal{G} / \operatorname{Stab}_{0}(A)$ of the orbit of $A \in \mathcal{S}$ is integral if and only if the Weinstein homomorphism $\pi_{1}\left(\operatorname{Ham}\left(\omega_{A}\right)\right) \rightarrow S^{1}$ is trivial (where $\operatorname{Stab}_{0}(A) \subset \mathcal{G}$ is the identity component of the stabilizer of $A$ and $\operatorname{Ham}\left(\omega_{A}\right)$ is the group of Hamiltonian diffeomorphisms).

The first three parts are proved in Section 2.2, while the statement concerning the Weinstein homomorphism is shown in Section 2.3.

In Section 3, we consider the restriction of the moment map $\mu$ for the action of the subgroup $\mathcal{T}=\operatorname{Map}_{0}\left(M, S^{1}\right) \subset \mathcal{G}$ of bundle isometries covering the identity on $M$. It turns out that the moment map sends a connection $A$ to the volume form $\omega_{A}^{n} / n$ !. In this way the problem of prescribing the volume of a symplectic structure can be seen in terms of moment map geometry.

As we explain in Section 3.1 one outcome of this is that when $b_{1}(M)=0$ the space of symplectic forms with fixed volume form is naturally a symplectic manifold. When $b_{1}(M) \neq 0$ this space carries a torus-fibration with fibres of dimension $b_{1}(M)$ whose total space is naturally a symplectic manifold.

In Section 3.2, we consider the problem of prescribing the volume form of a Kähler metric. This is the renowned Calabi conjecture, now of course Yau's theorem [Yau78]. Using the picture outlined above we show how the Calabi conjecture can be phrased as finding a zero of the moment map inside a complex group orbit. This puts the problem into the same framework as the Hitchin-Kobayashi correspondence (concerning Hermitian-Einstein connections) and the Donaldson-Tian-Yau conjecture (concerning Kähler metrics with constant scalar curvature).

The focus of this note is to explain the above geometric picture; no attempt is made here, however, to explore the potential applications. Both Sections 2 and 3 end with a brief discussion of some of these possible directions for future research (some more speculative than others!).

\section{The space of connections with symplectic curvature}

2.1. A note on conventions and notation. The introduction speaks of unitary connections in a Hermitian line bundle $L \rightarrow M$ over a compact $2 n$-dimensional manifold. In what follows, it will be more convenient to use the language of principal bundles. We write $p: P \rightarrow M$ for the principal $S^{1}$ bundle associated to $L$ and $\xi$ for the vector field generating the $S^{1}$-action on $P$. In this formalism, a unitary connection is a 1 -form $A \in \Omega^{1}(P, \mathbb{R})$ which is both $S^{1}$-invariant and satisfies $A(\xi)=\frac{1}{2 \pi}$. Then $\omega_{A} \in \Omega^{2}(M, \mathbb{R})$ is defined by the equation $p^{*} \omega_{A}=d A$. Note connection 1 -forms are more usually taken to be imaginary valued, but to prevent a proliferation of factors of $2 \pi i$ we have divided out by this at the beginning. Our conventions mean that the tangent space to the space of connections is identified with $\Omega^{1}(M, \mathbb{R})$ where 
$a \in \Omega^{1}(M, \mathbb{R})$ corresponds to an infinitesimal change of $p^{*} a$ in the connection 1-form $A$ and an infinitesimal change of $d a$ in $\omega_{A}$.

We write $\mathcal{S}$ for the set of connections in $P$ for which $\omega_{A}$ is symplectic. This is open in the space of all connections (for, say, the $C^{\infty}$ topology) and so again, $T_{A} \mathcal{S}=\Omega^{1}(M, \mathbb{R})$. Finally, in this language, unitary bundle isomorphisms are $S^{1}$-equivariant diffeomorphisms of $P$. The group $\mathcal{G}$ denotes the identity component of the group of all unitary bundle isomorphisms. Its Lie algebra $\operatorname{Lie}(\mathcal{G})$ is the algebra of $S^{1}$-invariant vector fields on $P$.

2.2. Symplectic structure and moment map. We begin by describing a symplectic structure on $\mathcal{S}$.

Definition 2. We define a 2 -form $\Omega$ on $\mathcal{S}$ by

$$
\Omega_{A}(a, b)=\frac{1}{(n-1) !} \int_{M} a \wedge b \wedge \omega_{A}^{n-1},
$$

for $a, b \in \Omega^{1}(M, \mathbb{R})=T_{A} \mathcal{S}$.

Proposition 3. The 2-form $\Omega$ is a symplectic form.

Proof. To prove non-degeneracy on $T_{A} \mathcal{S}$, let $J$ be an almost complex structure on $M$ compatible with $\omega_{A}$. The action of $J$ on $T M$ determines a dual action on $T^{*} M$ given by $(J a)(v)=-a(J v)$. For a real 1 -forms $a, b$ we have the following identity:

$$
\langle a, b\rangle \omega_{A}^{n}=n a \wedge J b \wedge \omega_{A}^{n-1}
$$

where the innerproduct $\langle a, b\rangle$ is that determined by $J$ and $\omega_{A}$. It follows that for any 1-form $a$ :

$$
\Omega_{A}(a, J a)=\frac{1}{n !} \int_{M}|a|^{2} \omega_{A}^{n},
$$

which is non-zero whenever $a$ is.

Next, we check $\Omega$ is closed. For this let $a, b, c \in \Omega^{1}(X, \mathbb{R})$, thought of as vector fields on $\mathcal{S}$. Then

$$
d \Omega(a, b, c)=a \cdot \Omega(b, c)+b \cdot \Omega(c, a)+c \cdot \Omega(a, b) .
$$

(The formula for general vector fields also includes terms with Lie brackets, but in our case these vanish since the vector fields $a, b, c$ are linear on the affine space of all connections and so commute.) Now,

$$
a \cdot \Omega(b, c)=\frac{1}{(n-2) !} \int_{M} b \wedge c \wedge d a \wedge \omega_{A}^{n-2} .
$$


Hence

$$
\begin{aligned}
d \Omega(a, b, c) & =\frac{1}{(n-2) !} \int_{M}(d a \wedge b \wedge c+d b \wedge c \wedge a+d c \wedge a \wedge b) \wedge \omega_{A}^{n-2} \\
& =\frac{1}{(n-2) !} \int_{M} d\left(a \wedge b \wedge c \wedge \omega_{A}^{n-2}\right) \\
& =0
\end{aligned}
$$

Referee claims $\Omega$ is exact. To show this they pick a reference connection $A_{0} \in \mathcal{S}$ and then set

$$
\Theta_{A}(a)=\frac{1}{(n+1)(n-1) !} \int_{M}\left(A-A_{0}\right) \wedge a \wedge \omega_{A}^{n-1} .
$$

Now $d \Theta(a, b)=a \cdot \Theta(b)-b \cdot \Theta(a)$. (The formula for general vector fields also includes a term with a Lie bracket, but in our case this vanishes since the vector fields $a, b$ are linear on the affine space of all connections and so commute.) Then

$$
\begin{aligned}
a \cdot \Theta(b)= & \frac{1}{(n+1)(n-1) !} \int_{M}\left(a \wedge b \wedge \omega_{A}^{n-1}+(n-1)\left(A-A_{0}\right) \wedge b\right. \\
& \left.\wedge d a \wedge \omega_{A}^{n-2}\right)
\end{aligned}
$$

In the equation for $d \Theta$ I seem to get then

$$
d \Theta(a, b)=\frac{2}{n+1} \Omega(a, b)+\frac{1}{(n+1)(n-2) !} \int_{M}\left(A-A_{0}\right) \wedge d(a \wedge b) \wedge \omega_{A}^{n-2}
$$

Now

$$
\left(A-A_{0}\right) \wedge d(a \wedge b) \wedge \omega_{A}^{n-2}=a \wedge b \wedge\left(\omega_{A}-\omega_{A_{0}}\right) \wedge \omega_{A}^{n-2}-d\left(\left(A-A_{0}\right) \wedge a \wedge b \wedge \omega_{A}^{n-2}\right) .
$$

So,

$\frac{1}{(n-1) !} \int_{M}\left(A-A_{0}\right) \wedge d(a \wedge b) \wedge \omega_{A}^{n-2}=\Omega(a, b)-\frac{1}{(n-1) !} \int_{M} a \wedge b \wedge \omega_{A_{0}} \wedge \omega_{A}^{n-2}$.

This means that I get

$$
d \Theta(a, b)=\Omega(a, b)-\frac{n-1}{n+1} \int_{M} a \wedge b \wedge \omega_{A_{0}} \wedge \omega_{A}^{n-2} .
$$

But I don't see why $d \Theta=\Omega$.

Next we turn to the group action. Recall that $\mathcal{G}$ is the identity component of the group of $S^{1}$-equivariant diffeomorphisms of $P$. This group acts by pull-back on connection 1-forms, preserving the set $\mathcal{S}$ and the symplectic structure $\Omega$. We now give a moment map for this action.

The Lie algebra $\operatorname{Lie}(\mathcal{G})$ is the space of $S^{1}$-invariant vector fields on $P$. Given a connection $A \in \Omega^{1}(P, \mathbb{R})$ and $\eta \in \operatorname{Lie}(\mathcal{G})$, the pairing $A(\eta)$ is an $S^{1}$-invariant function on $P$ and so descends to a function on $M$ which we also denote $A(\eta)$. 
Proposition 4. The map $\mu: \mathcal{S} \rightarrow \operatorname{Lie}(\mathcal{G})^{*}$ defined by

$$
\langle\mu(A), \eta\rangle=\frac{1}{n !} \int_{M} A(\eta) \omega_{A}^{n}
$$

is a $\mathcal{G}$-equivariant moment map for the action of $\mathcal{G}$ on $\mathcal{S}$.

Proof. Given $\eta \in \operatorname{Lie}(\mathcal{G})$, let $a_{\eta}$ be the vector field on $\mathcal{S}$ corresponding to the infinitesimal action of $\eta$. In other words, at $A \in \mathcal{S}, a_{\eta}$ is the 1-form given by

$$
\begin{aligned}
a_{\eta} & =\mathcal{L}_{\eta}(A) \\
& =\left(d \circ \iota_{\eta}+\iota_{\eta} \circ d\right) A \\
& =d(A(\eta))+\iota_{p_{*} \eta} \omega_{A},
\end{aligned}
$$

(where in the first two lines we have implicitly identified $a_{\eta}$ and $p^{*} a_{\eta}$ ). Let $b \in \Omega^{1}(M, \mathbb{R})$ be another vector field on $\mathcal{S}$. The identity to be proved is $b \cdot\langle\mu, \eta\rangle=\Omega\left(b, a_{\eta}\right)$.

We begin with the left-hand side. The vector field $b \in \Omega^{1}(M, \mathbb{R})$ on $\mathcal{S}$ corresponds to an infinitesimal change of $p^{*} b$ in $A$ and hence an infinitesimal change of $p^{*} b(\eta)=b\left(p_{*} \eta\right)$ in $A(\eta)$. Meanwhile, the infinitesimal change in $\omega_{A}$ is $d b$.

Hence,

$$
b \cdot\langle\mu, \eta\rangle=\int_{M}\left(\frac{1}{n !} b\left(p_{*} \eta\right) \omega_{A}^{n}+\frac{1}{(n-1) !} A(\eta) d b \wedge \omega_{A}^{n-1}\right) .
$$

To compute the right-hand side of the moment-map identity we have that, at $A \in \mathcal{S}$,

$$
\Omega\left(b, a_{\eta}\right)=\frac{1}{(n-1) !} \int_{M} b \wedge\left(d(A(\eta))+\iota_{p_{*} \eta} \omega_{A}\right) \wedge \omega_{A}^{n-1} .
$$

Next we use the following identity: on a $2 n$-dimensional manifold, given a 1 -form $\alpha$ and a 2 -form $\beta$ the $(2 n+1)$-form $\alpha \wedge \beta^{n}$ necessarily vanishes; hence, for any vector field $v$,

$$
0=\iota_{v}\left(\alpha \wedge \beta^{n}\right)=\alpha(v) \beta^{n}-n \alpha \wedge \iota_{v} \beta \wedge \beta^{n-1} .
$$

Putting $\alpha=b, \beta=\omega_{A}$ and $v=p_{*} \eta$, this gives

$$
\begin{aligned}
\Omega\left(b, a_{\eta}\right) & =\frac{1}{(n-1) !} \int_{M}\left(b \wedge d(A(\eta)) \wedge \omega_{A}^{n-1}+\frac{1}{n} b\left(p_{*} \eta\right) \omega_{A}^{n}\right), \\
& =\int_{M}\left(\frac{1}{(n-1) !} A(\eta) d b \wedge \omega_{A}^{n-1}+\frac{1}{n !} b\left(p_{*} \eta\right) \omega_{A}^{n}\right), \\
& =b \cdot\langle\mu, \eta\rangle .
\end{aligned}
$$

Finally, $\mathcal{G}$-equivariance follows immediately from the definition of $\mu$.

We remark that this picture is motivated by the well-known observation of Atiyah and Bott [AB83] that "curvature is a moment map". In [AB83], 
Atiyah and Bott consider unitary connections in bundles of arbitrary rank, but over a base with a fixed symplecitc form.

To complete the proof of Theorem 1 we show that the components of $\mathcal{S}$ are identified via $\mu$ with coadjoint orbits.

Lemma 5. The map $\mu: \mathcal{S} \rightarrow \operatorname{Lie}(\mathcal{G})^{*}$ embeds each component of $\mathcal{S}$ as a coadjoint orbit of $\mathcal{G}$.

Proof. We must show two things: firstly, that $\mu$ is injective; secondly that $\mathcal{G}$ acts transitively on the components of $\mathcal{S}$.

To prove injectivity of $\mu$, suppose that $A \neq A^{\prime}$. Then we can find a vector field $v$ on $M$ such that the $A^{\prime}$-horizontal lift $\eta$ of $v$ satisfies $A(\eta)>0$, hence $\langle\mu(A), \eta\rangle>0$. But $A^{\prime}(\eta)=0$ and so $\left\langle\mu\left(A^{\prime}\right), \eta\right\rangle=0$, hence $\mu(A) \neq \mu\left(A^{\prime}\right)$.

Next, we show that $\mathcal{G}_{0}$ acts transitively on the components of $\mathcal{S}$. Given $A \in \mathcal{S}$, let $\rho_{A}: \operatorname{Lie}(\mathcal{G}) \rightarrow T_{A} \mathcal{S}$ denote the infinitesimal action of $\mathcal{G}$ at $A$. We have already seen that

$$
\rho_{A}(\eta)=a_{\eta}=d(A(\eta))+\iota_{p_{*} \eta} \omega_{A} .
$$

First, we show that $\rho_{A}$ is surjective. Given $a \in \Omega^{1}(M, \mathbb{R})$, let $v$ be the $\omega_{A^{-}}$ dual vector field and let $\eta$ be the $A$-horizontal lift of $v$ to $P$. Then $\rho_{A}(\eta)=a$.

Now, given a path $A(t)$ in $\mathcal{S}$, let $v(t)$ be the vector field, which is $\omega_{A(t)^{-}}$ dual to $\frac{d A}{d t}(t)$ and let $\eta(t)$ be the $A(t)$-horizontal lift of $v(t)$ to $P$. The time-dependent vector field $\eta(t)$ integrates up to a path $g(t)$ in $\mathcal{G}_{0}$ with $g(0)$ the identity. By construction, $g(t) \cdot A(0)=A(t)$.

2.3. Integrality and the Weinstein homomorphism. We next turn to the question of whether or not the orbits of $\mathcal{S}$ are integral coadjoint orbits. It turns out that, up to taking a certain cover, the obstruction to this is a homomorphism $\pi_{1}\left(\operatorname{Ham}_{\omega_{A}}\right) \rightarrow S^{1}$, first introduced by Weinstein [Wei89].

We briefly recall the definition of an integral coadjoint orbit. For more details see, for example, [Kir04]. Given a Lie group $G$ with Lie algebra $\mathfrak{g}$, fix $f \in \mathfrak{g}^{*}$. We write $\operatorname{Stab}(f) \subset G$ for the stabilizer of $f$ under the coadjoint action and $\mathfrak{h}$ for the Lie algebra of the stabilizer. The linear map $f: \mathfrak{g} \rightarrow \mathbb{R}$ restricts to a Lie algebra homomorphism $f: \mathfrak{h} \rightarrow \mathbb{R}$. The orbit $\mathcal{O}_{f}$ of $f$ is called integral when the map $\mathfrak{h} \rightarrow \mathbb{R}$ is (up to a factor of $i$ ) the derivative of a homomorphism $w: \operatorname{Stab}(f) \rightarrow S^{1}$.

This condition implies the existence of a principal circle bundle $Q \rightarrow \mathcal{O}_{f}$ which carries a connection whose curvature is the symplectic form on $\mathcal{O}_{f}$; moreover the symplectic action of $G$ on $\mathcal{O}_{f}$ lifts to a connection-preserving action on $Q$. To see this, first note that $G \rightarrow \mathcal{O}_{f}$ is the total space of a principal $\operatorname{Stab}(f)$-bundle. The representation $w$ gives rise to an associated principal $S^{1}$-bundle

$$
Q=G \times_{w} S^{1} \rightarrow \mathcal{O}_{f}
$$


The action of $G$ on itself by left-multiplication commutes with that of rightmultiplication by $\operatorname{Stab}(f)$ and so $G$ acts on $Q$ covering the action on $\mathcal{O}_{f}$. At a point $q \in Q$ the derivative of this action gives a map $L_{q}: \mathfrak{g} \rightarrow T_{q} Q$ whose image is transverse to the tangent space to the $S^{1}$-fibre through $q$. So $L_{q}(\mathfrak{g}) \subset T_{q} Q$ defines a connection, in the sense of a horizontal distribution in $Q$, which one checks is $S^{1}$-invariant and $G$-invariant and whose curvature is the symplectic form on $\mathcal{O}_{f}$.

In our situation, when considering integrality it is easier to work not with the coadjoint orbit itself, but instead with $\widetilde{\mathcal{O}}_{f} \cong G / \operatorname{Stab}_{0}(f)$ where $\operatorname{Stab}_{0}(f)$ is the identity component of $\operatorname{Stab}(f)$. This space is a cover of $\mathcal{O}_{f}$ with fibre $\operatorname{Stab}(f) / \operatorname{Stab}_{0}(f)$ and inherits a symplectic structure by pulling back that from $\mathcal{O}_{f}$. We call $\widetilde{\mathcal{O}}_{f}$ integral when the map $\mathfrak{h} \rightarrow \mathbb{R}$ is (up to a factor of $i$ ) the derivative of a homomorphism $w: \operatorname{Stab}_{0}(f) \rightarrow S^{1}$. Again, this condition implies the existence of a principal circle bundle $\widetilde{Q} \rightarrow \widetilde{\mathcal{O}}_{f}$ with connection whose curvature is the symplectic form. The action of $G$ also lifts to a connection-preserving action on $\widetilde{Q}$.

Accordingly, we next investigate the identity component $\operatorname{Stab}_{0}(A)$ of the stabilizer $\operatorname{Stab}(A) \subset \mathcal{G}$ of a point $A \in \mathcal{S}$. For an alternative exposition of the following, see Weinstein's article [Wei89].

We start from the short exact sequence

$$
1 \rightarrow \operatorname{Map}_{0}\left(M, S^{1}\right) \rightarrow \mathcal{G} \rightarrow \operatorname{Diff}_{0}(M) \rightarrow 1
$$

(where the subscripts 0 denote the identity components.)

Lemma 6 (Weinstein [Wei89]). Restricting this short exact sequence to $\operatorname{Stab}_{0}(A)$ gives the short exact sequence

$$
1 \rightarrow S^{1} \rightarrow \operatorname{Stab}_{0}(A) \rightarrow \operatorname{Ham}\left(\omega_{A}\right) \rightarrow 1
$$

where $S^{1} \subset \operatorname{Map}_{0}\left(M, S^{1}\right)$ are the constant gauge transformations.

Proof. First note that the restriction of the map $\mathcal{G} \rightarrow \operatorname{Diff}_{0}(M)$ to $\operatorname{Stab}_{0}(A)$ certainly takes values in $\omega_{A}$-symplectomorphisms. To verify that the image lies in $\operatorname{Ham}\left(\omega_{A}\right)$, recall the formula $(2.1)$ for the infinitesimal action $\rho_{A}(\eta)$ of $\eta \in \operatorname{Lie}(\mathcal{G})$ at $A$ given above. From this it follows that $\eta \in \operatorname{Lie}(\operatorname{Stab}(A))$ if and only if $p_{*} \eta$ is a Hamiltonian vector field with Hamiltonian $-A(\eta)$. Integrating this result we see that the image of $\operatorname{Stab}_{0}(A)$ lies in $\operatorname{Ham}\left(\omega_{A}\right)$.

Next we check that the map $\pi: \operatorname{Stab}_{0}(A) \rightarrow \operatorname{Ham}\left(\omega_{A}\right)$ is surjective. Given a $\omega_{A}$-Hamiltonian vector field $v$ on $M$ with Hamiltonian $h$ we write $v^{b}$ for the $A$-horizontal lift of $v$. Then the vector field $\eta=v^{b}-h \frac{\partial}{\partial \theta}$ on $P$ is $S^{1}$-invariant, hence in $\operatorname{Lie}(\mathcal{G})$ and $\rho_{A}(\eta)=0$. So $\eta \in \operatorname{Lie}\left(\operatorname{Stab}_{A}\right)$ and $\pi_{*}(\eta)=v$, meaning $\pi_{*}$ is surjective. Integrating this shows that $\pi: \operatorname{Stab}_{0}(A) \rightarrow \operatorname{Ham}\left(\omega_{A}\right)$ is surjective. 
The kernel of $\pi$ is $\operatorname{Stab}_{0}(A) \cap \operatorname{Map}_{0}\left(M, S^{1}\right)$. Given $f: M \rightarrow S^{1}$, the corresponding change in $A$ is $f d\left(f^{-1}\right)$. Hence, $\operatorname{ker} \pi=S^{1}$ is the constants, and the short exact sequence for $\mathcal{G}$ restricts to $\operatorname{Stab}_{0}(A)$ as claimed.

Given $A \in \mathcal{S}$ the moment map at $A$ restricts to give a Lie algebra homomorphism

$$
\mu(A): \operatorname{Lie}(\operatorname{Stab}(A)) \rightarrow \mathbb{R} .
$$

The kernel of this map is an ideal $I \subset \operatorname{Lie}(\operatorname{Stab}(A))$; moreover, the inclusion $S^{1} \subset \operatorname{Stab}(A)$ determines a copy of $\mathbb{R} \subset \operatorname{Lie}\left(\operatorname{Stab}_{A}\right)$, which is mapped isomorphically onto $\mathbb{R}$ by $\mu$. It follows that the derivative of $\operatorname{Stab}(A) \rightarrow$ $\operatorname{Ham}(A)$ identifies $I \cong \operatorname{HVect}\left(\omega_{A}\right)$ and so there is a splitting

$$
\operatorname{Lie}(\operatorname{Stab}(A)) \cong \mathbb{R} \oplus \operatorname{HVect}\left(\omega_{A}\right)
$$

into a direct sum of ideals.

Alternatively, one can see this splitting via an isomorphism

$$
C^{\infty}(M, \mathbb{R}) \cong \operatorname{Lie}(\operatorname{Stab}(A)) .
$$

The isomorphism sends a function $h$ to the vector field $v_{h}^{b}-h \frac{\partial}{\partial \theta}$ where $v_{h}$ is the Hamiltonian vector field corresponding to $h$. This map is an isomorphism of Lie algebras where $C^{\infty}(M, \mathbb{R})$ is endowed with the Poisson bracket defined by $\omega_{A}$. Now the above splitting (2.3) corresponds to the constant functions plus those with $\omega_{A}$-mean value zero.

Using left-multiplication we can view the splitting (2.3) as defining a connection on the principal $S^{1}$-bundle $\operatorname{Stab}_{0}(A) \rightarrow \operatorname{Ham}\left(\omega_{A}\right)$. Because the horizontal subspace (the HVect $\left(\omega_{A}\right)$ summand) is a Lie sub-algebra of $\operatorname{Lie}(\operatorname{Stab}(A))$, this connection is flat.

Lemma 7. The holonomy of the flat connection in $\operatorname{Stab}_{0}(A) \rightarrow \operatorname{Ham}\left(\omega_{A}\right)$ is the Weinstein homomorphism [Wei89],

$$
w: \pi_{1}\left(\operatorname{Ham}\left(\omega_{A}\right)\right) \rightarrow S^{1} .
$$

Proof. One definition of the Weinstein homomorphism is the following. Let $[\phi] \in \pi_{1}\left(\operatorname{Ham}\left(\omega_{A}\right)\right)$ where $\phi$ is generated by the Hamiltonian function $H: M \times S^{1} \rightarrow \mathbb{R}$. We assume moreover that each $H_{t}$ has mean-value zero, i.e, for each $t \in S^{1}, \int_{M} H_{t} \omega_{A}^{n}=0$. Pick a point $x \in M$ and a disc $D$ bounding the loop $\phi_{t}(x)$. The value of the Weinstein homomorphism on $[\phi]$ is defined to be the image $z \in S^{1}=\mathbb{R} / \mathbb{Z}$ of the real number

$$
\tilde{z}=\int_{D} \omega_{A}-\int_{0}^{1} H_{t}\left(\phi_{t}(x)\right) d t
$$

Such a disc can always be found and different discs change $\tilde{z}$ by an integer given by integrating $\omega_{A}$ over a sphere in $M$, and hence do not change $z \in S^{1}$. To complete the definition of the Weinstein homomorphism, one checks that $z$ does not depend on the choice of $x$ or on the representative of the homotopy 
class $[\phi]$. Then $w([\phi])=z$ gives a well-defined map $\pi_{1}\left(\operatorname{Ham}\left(\omega_{A}\right)\right) \rightarrow S^{1}$, which one checks is a homomorphism.

We now show that this agrees with the holonomy of the flat connection in the principle $S^{1}$-bundle $\operatorname{Stab}_{0}(A) \rightarrow \operatorname{Ham}\left(\omega_{A}\right)$. Recall that the flat connection is defined by setting the horizontal lift of $v \in \operatorname{HVect}\left(\omega_{A}\right)$ with mean-value zero Hamiltonian $h$ to be the vector field $\hat{v} \in \operatorname{Lie}(\operatorname{Stab}(A))$ given by

$$
\hat{v}=v^{b}-h \frac{\partial}{\partial \theta} \text {. }
$$

Now let $\phi$ be a loop in $\operatorname{Ham}\left(\omega_{A}\right)$ generated by $H$ as before. We now lift $\phi$ to a path in $\operatorname{Stab}_{0}(A)$ via the flat connection. Let $v_{t}$ be the vector field generating $\phi$ and $\hat{v}_{t}$ the lift of $v_{t}$ to $P \rightarrow M$ given by

$$
\hat{v}_{t}=v_{t}^{b}-H_{t} \frac{\partial}{\partial \theta} \text {. }
$$

The lift of the loop $\phi$ to $\operatorname{Stab}_{0}(A)$ is the flow of $\hat{v}_{t}$.

Now the holonomy along $\phi$ is a constant gauge transformation and so to compute it, it suffices to find its action at a single point. Pick $x \in M$ and $p \in P$ above $x$. The flow $\gamma:[0,1] \rightarrow P$ of $\hat{v}_{t}$ starting at $p$ covers the loop $\phi_{t}(x)$ in $M$; since $\gamma(0)$ and $\gamma(1)$ both lie over $x$ they differ by $z \in S^{1}$, which is the holonomy along $\phi$ that we seek. To see why this is the same $z$ as before, note that the first term in the formula (2.4) for $\tilde{z}$ gives the holonomy of $A$ around $\phi_{t}(p)$, hence the contribution of the first term in the above formula (2.5) for $\hat{v}_{t}$ while the second integral in (2.4) gives the contribution of the second term in (2.5).

Remark 8. One can interpret this result as giving an alternative definition of the Weinstein homomorphism in which:

(1) The independence of $z$ on the point $x$ and the representative $\phi$ of the class $[\phi]$ is immediate, by virtue of the definition as a holonomy representation.

(2) There is no need to assume the existence of a disc $D$ bounding $\phi_{t}(x)$ in order to define $w$ as a holonomy representation; one only needs the disc to write down the formula (2.4). Such a disc does indeed exist, but the proof is in general deep, relying on the theory of pseudoholomorphic curves. The definition of $w$ in terms of holonomy given here is purely "classical", answering a comment of McDuff, see footnote 3 of [McD10] (at least in the case $[\omega]$ is an integral class).

Proposition 9. Given $A \in \mathcal{S}$, the cover $\widetilde{\mathcal{O}}_{A} \cong \mathcal{G} / \operatorname{Stab}_{0}(A)$ of the corresponding coadjoint orbit $\mathcal{O}_{A}$ of $\mathcal{G}$ is integral if and only if the Weinstein homomorphism $w: \pi_{1}\left(\operatorname{Ham}\left(\omega_{A}\right)\right) \rightarrow S^{1}$ is trivial.

Proof. The cover $\widetilde{\mathcal{O}}_{A}$ is integral precisely when the kernel of the homomorphism $\mu(A): \operatorname{Lie}(\operatorname{Stab}(A)) \rightarrow \mathbb{R}$ integrates up to a subgroup of $\operatorname{Stab}_{0}(A)$. 
In our case, this kernel defines the horizontal space of the flat connection whose holonomy is $w$. So the orbit is integral if and only if parallel transport identifies all the $S^{1}$-fibres of $\operatorname{Stab}_{0}(A) \rightarrow \operatorname{Ham}\left(\omega_{A}\right)$. This happens precisely when the holonomy is trival.

On the one hand, there are examples of symplectic manifolds for which the Weinstein homomorphism is trivial. Indeed, for a surface of genus at least one, the Hamiltonian group is even contractible. On the other hand, there are also plenty of manfiolds for which the Weinstein homomorphism is non-trivial; the simplest being $S^{2}$. To see this, restrict the short exact sequence (2.2) to the subgroup $\mathrm{SO}(3) \subset$ Ham to obtain the sequence

$$
1 \rightarrow S^{1} \rightarrow \mathrm{U}(2) \rightarrow \mathrm{SO}(3) \rightarrow 1 .
$$

The flat connection corresponds to the Lie algebra isomorphism $\mathfrak{u}(2) \cong$ $\mathfrak{s u}(2) \oplus i \mathbb{R}$; its holonomy is non-trivial and gives the standard isomorphism

$$
\mathrm{U}(2) \cong \mathrm{SU}(2) \times_{ \pm 1} S^{1} .
$$

Similiar remarks apply to $\mathbb{C P}^{n}$ with the Fubini-Study metric and, more generally to certain toric varieties. See the recent survey article of McDuff [McD10] for more on this subject.

2.4. Further questions. Given a subgroup $\mathcal{H} \subset \operatorname{Diff}_{0}(M)$, the preimage under $\mathcal{G} \rightarrow \operatorname{Diff}_{0}(M)$ is a subgroup $\mathcal{H}^{\prime} \subseteq \mathcal{G}$ which inherits a Hamiltonian action on $\mathcal{S}$. The moment-map $\mu^{\prime}$ for the action of $\mathcal{H}^{\prime}$ is simply the projection of $\mu$ under $\operatorname{Lie}(\mathcal{G})^{*} \rightarrow \operatorname{Lie}\left(\mathcal{H}^{\prime}\right)^{*}$. One might look for zeros of $\mu^{\prime}$ in the hope that they give symplectic structures which respect in some way the additional geometry imposed in passing from $\operatorname{Diff}_{0}(M)$ to $\mathcal{H}$.

We explore this idea in the next section in its most extreme form, when $\mathcal{H}=1$ is the trivial group. This leads to the problem of prescribing the volume form of a symplectic structure. In a separate article [Fin11] we exploit this same idea for certain manifolds $M$ and subgroups $\mathcal{H}$. The manifolds in question are $S^{2}$-bundles over four-manifolds and in this way we give a moment-map interpretation of the anti-self-dual Einstein equations for a Riemannian metric on a four-manifold. Besides these two situations, however, there are many other possibilities one could study and it would be interesting to see more examples.

We close this section with a speculative remark. The above picture associates to each isotopy class of symplectic forms in $c_{1}(L)$ a certain coadjoint orbit of $\mathcal{G}$. On the one hand, distinguishing isotopy classes of symplectic forms is a central problem in symplectic topology; on the other hand, classifying coadjoint orbits is a central problem in the theory of infinitedimensional Lie groups. One might hope that Theorem 1 opens up the path for a transfer of ideas between these two areas. 
An important approach to the study of coadjoint orbits is the celebrated "orbit method" (see, for example, the text of Kirillov [Kir04]). For the group $\mathcal{G}$, perhaps the first case to consider would be a surface of genus at least one. There, the corresponding coadjoint orbit is integral. Moreover, as we will see in the following section, it comes with a natural isotropic fibration whose infinite-dimensional fibres fail to be coisotropic by a finitedimensional discrepancy (see Remark 14). Thus we have in place more-orless the initial data required by geometric quantisation. This still leaves, of course, the principal difficulty of what should play the rŹle of the "squareintegrable sections" of the prequantum line bundle, since the base is infinitedimensional. Exactly how to quantize such a coadjoint orbit is, in my opinion at least, an interesting and difficult question.

\section{Prescribing the volume form of a symplectic structure}

Given a Hamiltonian action of a group $G$ with a moment map $\mu$ taking values in $\mathfrak{g}^{*}$, the action of a sub-group $H \subset G$ has moment map given by composing $\mu$ with the projection $\mathfrak{g}^{*} \rightarrow \mathfrak{h}^{*}$. In this section, we apply this observation to the action of the subgroup of bundle isometries of $L \rightarrow M$, which cover the identity.

3.1. Purely symplectic case. Of course, $\mathcal{T}=\operatorname{Map}\left(M, S^{1}\right)$ and so $\operatorname{Lie}(\mathcal{T})=C^{\infty}(X, \mathbb{R})$. (One normally uses imaginary valued functions here but again we have multiplied by $-2 \pi i$ throughout.) By integrating against top-degree forms, we can identify $\Omega^{2 n}(M, \mathbb{R})$ with a subset of $\operatorname{Lie}(\mathcal{T})^{*}$. With this understood, we have the following result, which is an immediate corollary of Proposition 4.

Proposition 10. There is an equivariant moment map $\nu: \mathcal{S} \rightarrow \operatorname{Lie}(\mathcal{T})^{*}$ for the action of $\mathcal{T}$ on $\mathcal{S}$ given by $\nu(A)=\omega_{A}^{n} / n$ !.

(Strictly speaking, above we only mentioned the invariance of the symplectic form $\Omega$ on $\mathcal{S}$ for the identity component $\mathcal{G}$ of the group of bundle isometries, but it is clear from the definition that $\Omega$ is invariant under the action of all bundle isometries, which cover orientation preserving diffeomorphisms of $M$, a group which contains $\mathcal{T}$, unlike $\mathcal{G}$ which only contains null-homotopic maps $M \rightarrow S^{1}$.)

So prescribing the volume of a symplectic structure in $c_{1}(L)$ can be seen as finding a zero of a moment map. More precisely, since $\mathcal{T}$ is abelian, the coadjoint action is trivial and so we can equally use $\nu-\theta$ as a moment map for any $\theta \in \operatorname{Lie}(\mathcal{T})^{*}$. Given a volume form $\theta \in \Omega^{2 n}(M, \mathbb{R})$ with $[\theta]=\frac{1}{n !} c_{1}(L)^{n}$, the equation for $A \in \mathcal{S}$ given by $\omega_{A}^{n} / n !=\theta$ is the same as finding a zero of the moment map $\nu-\theta$.

Given such a $\theta$, we next turn to the symplectic reduction $\nu^{-1}(\theta) / \mathcal{T}$. By standard theory this is a symplectic manifold (of infinite dimension). To 
describe it we write $\mathcal{X}_{\theta}$ for the space of symplectic forms $\omega \in c_{1}(L)$ with $\omega^{n} / n !=\theta$.

Proposition 11. If $b_{1}(M)=0$ then $\mathcal{X}_{\theta}=\nu^{-1}(\theta) / \mathcal{T}$. In particular, the space of symplectic forms with fixed volume form is naturally a symplectic manifold.

In general there is a submersion $\nu^{-1}(\theta) / \mathcal{T} \rightarrow \mathcal{X}_{\theta}$ with fibres isomorphic to $H^{1}(M, \mathbb{R}) / H^{1}(M, \mathbb{Z})$. The restriction of the symplectic structure to these fibres is identified with the 2-form on $H^{1}(M, \mathbb{R})$ defined by

$$
(\alpha, \beta) \mapsto \frac{1}{(n-1) !} \int_{M} \alpha \wedge \beta \wedge c_{1}(L)^{n-1} .
$$

Proof. We begin with the following standard fact. Given a symplectic form $\omega \in c_{1}(L)$, write $\mathcal{S}_{\omega} \subset \mathcal{S}$ for the set of unitary connections $A$ for which $\omega_{A}=\omega$. Then $\mathcal{S}_{\omega} / \mathcal{T}$ can be identified with $H^{1}(M, \mathbb{R}) / H^{1}(M, \mathbb{Z})$.

More precisely, given $A_{0} \in \mathcal{S}_{\omega}$, any other connection $A \in \mathcal{S}_{\omega}$ is of the form $A=A_{0}+\frac{i}{2 \pi} a$ for a closed 1-form $a$. There is thus a surjection $c: \mathcal{S}_{\omega} \rightarrow H^{1}(M, \mathbb{R})$ given by $c(A)=[a]$. Now $\mathcal{T}=\operatorname{Map}\left(M, S^{1}\right)$ acts on $H^{1}(M, \mathbb{R})$, the action of $f \in \mathcal{T}$ on $H^{1}(M, \mathbb{R})$ is by addition of $\frac{1}{2 \pi i}\left[f d\left(f^{-1}\right)\right] \in$ $H^{1}(M, \mathbb{Z})$. With this action understood, $c$ is $\mathcal{T}$-equivariant. Since any element of $H^{1}(M, \mathbb{Z})$ can be written in as $\frac{1}{2 \pi i}\left[f d\left(f^{-1}\right)\right]$ for some $f \in \mathcal{T}$, the map $c$ descends to an identification $\mathcal{S}_{\omega} / \mathcal{T} \rightarrow H^{1}(M, \mathbb{R}) / H^{1}(M, \mathbb{Z})$.

The group $\mathcal{T}$ is abelian, so its orbits in $\mathcal{S}$ are isotropic and hence the restriction of the symplectic form $\Omega$ on $\mathcal{S}$ to $\mathcal{S}_{\omega}$ descends to a 2 -form on $\mathcal{S}_{\omega} / \mathcal{T} \cong H^{1}(M, \mathbb{R}) / H^{1}(M, \mathbb{Z})$. It follows from the definition of $\Omega$ that the 2 -form is identified with the 2 -form on $H^{1}(M, \mathbb{R})$ given by

$$
(\alpha, \beta) \mapsto \frac{1}{(n-1) !} \int_{M} \alpha \wedge \beta \wedge c_{1}(L)^{n-1} .
$$

The result follows from these two observations applied fibrewise to the map $\nu^{-1}(\theta) \rightarrow \mathcal{X}_{\theta}$, which sends each connection $A$ to its curvature $\omega_{A}$.

Remark 12. When $b_{1}(M)=0$, the symplectic structure on $\mathcal{X}_{\theta}$ can be seen directly (and with no need for the condition that the fixed choice of symplectic class be integral). The tangent space at a point $\omega \in \mathcal{X}_{\theta}$ is the space of exact 2-forms $\gamma$ such that $\omega^{n-1} \wedge \gamma=0$. We now define a skew pairing $\Theta$ on $T_{\omega} \mathcal{X}_{\theta}$ by

$$
\Theta\left(\gamma, \gamma^{\prime}\right)=\frac{1}{(n-1) !} \int_{M} a \wedge a^{\prime} \wedge \omega^{n-1},
$$

where $a, a^{\prime}$ are 1 -forms with $d a=\gamma, d a^{\prime}=\gamma^{\prime}$. If $\tilde{a}$ is another 1 -form with $d \tilde{a}=\gamma$, then $d(a-\tilde{a})=0$ and so, since $b_{1}(M)=0$, we can write $a-\tilde{a}=d f$ for some function $f$. Hence,

$$
\int_{M}(a-\tilde{a}) \wedge a^{\prime} \wedge \omega^{n-1}=-\int_{M} f d a^{\prime} \wedge \omega^{n-1},
$$


which vanishes since $d a^{\prime} \wedge \omega^{n-1}=\gamma^{\prime} \wedge \omega^{n-1}=0$. It follows that $\Theta\left(\gamma, \gamma^{\prime}\right)$ does not depend on the choice of $a$ or $a^{\prime}$.

When the fixed symplectic class $[\omega]=c_{1}(L)$ is integral, $\Theta$ is precisely the 2 -form, which arises from the identification $\nu^{-1}(\theta) / \mathcal{T} \cong \mathcal{X}_{\theta}$. It follows from the general theory that $\Theta$ is closed and non-degenerate, something which one can verify directly from the definition.

Remark 13. Still under the assumption that $b_{1}(M)=0$, note that the group $\operatorname{Diff}(M, \theta)$ of volume-preserving diffeomorphisms acts on the symplectic manifold $\mathcal{X}_{\theta}$. This action is Hamiltonian in the sense that the infinitesimal action of a single divergence-free vector field $u$ is a Hamiltonian vector field on $\mathcal{X}_{\theta}$. To define a Hamiltonian $h: \mathcal{X}_{\theta} \rightarrow \mathbb{R}$ for the action of $u$ note that $L_{u} \theta=0$ so $\iota_{u} \theta$ is a closed $(2 n-1)$-form. Since $b_{1}(M)=b_{2 n-1}(M)=0$, we can write $\iota_{u} \theta=d \beta$ for some $(2 n-2)$-form $\beta$. We define the function $h$ by

$$
h(\omega)=-\int_{M} \beta \wedge \omega .
$$

Given a tangent vector $\gamma \in T_{\omega} \mathcal{X}_{\theta}$, i.e., an exact 2-form $\gamma=d a$ with $\gamma \wedge$ $\omega^{n-1}=0$, then the corresponding infinitesimal change in $h$ is given by

$$
\gamma \cdot h=-\int_{M} \beta \wedge \gamma=-\int_{M} \beta \wedge d a=\int_{M} \iota_{u} \theta \wedge a .
$$

On the other hand, the infinitesimal action of $u$ at $\omega \in \mathcal{X}_{\theta}$ is $\gamma_{u}=d\left(\iota_{u} \omega\right)$ and so

$$
\Theta\left(\gamma_{u}, \gamma\right)=\frac{1}{(n-1) !} \int_{M} \iota_{u} \omega \wedge a \wedge \omega^{n-1}=\int \iota_{u} \theta \wedge a .
$$

Hence, $h$ is a Hamiltonian for the action of $u$.

Of course the Hamiltonian $h$ is uniquely determined only up to the addition of a constant. This is reflected in our description of $h$ by the freedom in the choice of $\beta$; adding a closed $(2 n-2)$-form to $\beta$ does not alter $d \beta=\iota_{u} \theta$ but changes $h$ by a constant. Writing down a moment map for the action amounts to choosing these constants consistently. The choices involved suggest that this cannot be done in such a way as to give an equivariant moment map.

Remark 14. As mentioned above, $\mathcal{T}$ is abelian and so the $\mathcal{T}$-orbits in $\mathcal{S}$ are isotropic. It follows from the standard theory of symplectic reduction that the fibres of the moment map $\nu$ are coisotropic and, moreover, given $A \in \mathcal{S}$, the tangent space to the fibre of $\nu$ through $A$ is the symplectic complement of the tangent space to the $\mathcal{T}$-orbit through $A$. When $M$ is a surface, the isotropic fibration of $\mathcal{S}$ given by the $\mathcal{T}$-orbits is close to being a Lagrangian fibration. To see this, note that for a surface, volume forms and symplectic forms are the same thing. Now in the proof of Proposition 11 we saw that the codimension of $\mathcal{T} \cdot A$ in $\nu^{-1}\left(\omega_{A}\right)$ is $b_{1}(M)$. So for $S^{2}$ the $\mathcal{T}$-orbits give a Lagrangian fibration of $\mathcal{S}$, while for higher genus surfaces 
this infinite-dimensional isotropic fibration fails to be Lagrangian only by a finite-dimensional discrepancy.

3.2. The Kähler case. This point of view has additional use when $M$ is a complex manifold. Recall that the Calabi conjecture (now, of course, Yau's theorem [Yau78]) states that given a Kähler class $\kappa \in H^{2}(M, \mathbb{R})$ and volume-form $\theta$ on $M$ with total volume $\frac{1}{n !} \int_{M} \kappa^{n}$ there is a unique Kähler metric $\omega \in \kappa$ with $\omega^{n} / n !=\theta$.

(An alternative and perhaps more usual formulation of the Calabi conjecture is that given any closed real $(1,1)$-form $\rho$ representing $2 \pi c_{1}(M)$ there is a unique Kähler metric $\omega \in \kappa$ whose Ricci form is $\rho$. Passing between these two statements is a standard procedure in Kähler geometry: the volume form of a Kähler metric is at the same time a Hermitian metric on the anticanonical bundle $K^{*}$ since $\Lambda^{n, n} \cong K \otimes \bar{K}^{*} ;$ moreover, the Ricci form $\rho$ is, up to a factor of $i$, the curvature of $K^{*}$ with this Hermitian structure. So prescribing the volume form of a Kähler metric is the same thing as prescribing its Ricci curvature. See, for example, the introductory text [Tia00].)

When $\kappa=c_{1}(L)$ is the first Chern class of a holomorphic line bundle, we can reformulate the Calabi conjecture as the search for the zero of a moment map in a complex group orbit, in a manner analogous to the HitchinKobayashi correspondence [Don85, UY86] or the Donaldson-Tian-Yau conjecture concerning existence of constant scalar curvature Kähler metrics (as outlined in, for example, [Don02]).

To describe this we first restrict attention to the subspace $\mathcal{S}^{1,1} \subset \mathcal{S}$ of unitary connections in $L \rightarrow M$ whose curvature is a positive $(1,1)$-form on the complex manifold $M$. The complex structure $J$ on $M$ makes $\mathcal{S}^{1,1}$ into a Kähler manifold. To see this notice that the endomorphism $a \mapsto J a$ of $\Omega^{1}(M, \mathbb{R})$ makes $\mathcal{S}$ into an almost complex manifold. Given $A \in \mathcal{S}^{1,1}$, $a \in T_{A} \mathcal{S}^{1,1}$ if and only if $\bar{\partial}\left(a^{0,1}\right)=0$. Since $(J a)^{0,1}=-i a^{0,1}$ it follows that $\mathcal{S}^{1,1}$ is an almost complex submanifold of $\mathcal{S}$.

To show that this almost complex structure is integrable we use the standard identification of the space of unitary connections having curvature of type $(1,1)$ with the space of holomorphic structures on the line bundle $L \rightarrow M$. The identification sends a unitary connection $A$ to the $\bar{\partial}-$ operator given by the $(0,1)$-component of $A$. The $\bar{\partial}$-operator is integrable precisely because $\omega_{A}$ is $(1,1)$. A unitary connection is determined by its $(0,1)$-component and, conversely, every integrable $\bar{\partial}$-operator can be completed in a unique way to a unitary connection with $(1,1)$ curvature (see, e.g., [GH94]). In this way we identify $\mathcal{S}^{1,1}$ with the open subset of integrable $\bar{\partial}$-operators whose curvatures are in fact Kähler forms. Under this identification, the almost complex structure described in the preceding paragraph is identified with the natural holomoprhic structure on the space of integrable $\bar{\partial}$-operators. 
The symplectic structure $\Omega$ on $\mathcal{S}$ restricts to a Kähler metric on $\mathcal{S}^{1,1}$ : given $A \in \mathcal{S}^{1,1}, \omega_{A}$ and $J$ pair to give a Riemannian metric $g_{A}$ on $M$; now $\Omega_{A}(a, J a)=\|a\|^{2}$ is the $L^{2}\left(g_{A}\right)$-norm of $a$ and so the restriction of $\Omega$ to $\mathcal{S}^{1,1}$ pairs with the complex structure to give a Kähler metric on $\mathcal{S}^{1,1}$.

This whole setup is, of course, reminiscent of the moment-map description of the Hitchin-Kobayashi correspondence. There one starts with a Hermitian vector bundle (of arbitrary rank) $E \rightarrow M$ and considers the space $\mathcal{A}^{1,1}$ of all unitary connections with $(1,1)$-curvature or, equivalently, all integrable $\bar{\partial}$-operators. The key difference is that for the Hitchin-Kobayashi correspondence the symplectic structure on $\mathcal{A}^{1,1}$ is defined via a fixed choice of Kähler metric on $M$. In our situation, however, the Kähler form $\omega_{A}$ on $M$ depends on the unitary connection $A \in \mathcal{S}^{1,1}$ and the symplectic structure on $\mathcal{S}^{1,1}$ is different from that in the Hitchin-Kobayashi correspondence.

While the whole group $\mathcal{G}$ does not act by Kähler isometries on $\mathcal{S}^{1,1}$ (since the induced action on $M$ does not preserve $J$ ) the $\operatorname{subgroup} \mathcal{T}=\operatorname{Map}\left(M, S^{1}\right)$ does. The action extends, at least locally, to a holomorphic (though not isometric) action of the complexification $\mathcal{T}^{\mathbb{C}}=\operatorname{Map}\left(M, \mathbb{C}^{*}\right)$. This is most easily seen by considering $\mathcal{S}^{1,1} \subset \mathcal{H}$ as an open set in the space of integrable $\bar{\partial}$-operators in $L$. Now $\mathcal{T}^{\mathbb{C}}$ acts on $\mathcal{H}$ by pulling back. Note this is not the same as pulling back the corresponding unitary connection by an element of $\mathcal{T}^{\mathbb{C}}$, since this does not preserve the property of being unitary. In terms of connections, the action of $f \in \operatorname{Map}\left(M, \mathbb{C}^{*}\right)$ on $A$ is given by

$$
f \cdot A=A+f \bar{\partial}\left(f^{-1}\right)-\bar{f} \partial\left(\bar{f}^{-1}\right) .
$$

In particular, given a function $\phi \in C^{\infty}(M, \mathbb{R})$, the action of $f=e^{\phi}$ is

$$
e^{\phi} \cdot A=A+\partial \phi-\bar{\partial} \phi
$$

and hence

$$
\omega_{e^{\phi} \cdot A}=\omega_{A}+\frac{i}{2 \pi} \bar{\partial} \partial \phi
$$

From this formula it is clear that the $\mathcal{T}^{\mathbb{C}}$-orbit of $A \in \mathcal{S}^{1,1}$ leaves the open set $\mathcal{S}^{1,1} \subset \mathcal{H}$. Indeed $e^{\phi} \cdot A$ remains in $\mathcal{S}^{1,1}$ precisely when $\phi$ is a Kähler potential for $\omega_{A}$. Nonetheless this calculation proves the following result.

Lemma 15. Fix $A_{0} \in \mathcal{S}^{1,1}$. The map $A \mapsto \omega_{A}$ gives a surjection from $\left(\mathcal{T}^{\mathbb{C}} \cdot A_{0}\right) \cap \mathcal{S}^{1,1}$ to the space of Kähler metrics in $c_{1}(L)$.

From here we see that the Calabi conjecture fits into the general framework of moment maps in Kähler geometry. Namely, finding a Kähler form in $c_{1}(L)$ with volume form $\theta$ is the same as finding a zero of the moment map $\nu-\theta$ in a given complex orbit $\left(\mathcal{T}^{\mathbb{C}} \cdot A\right) \cap \mathcal{S}^{1,1}$.

We recall a little of the general set-up alluded to here. The starting point is the action of a Lie group $G$ by holomorphic isometries on a Kähler manifold $X$, along with an equivariant moment map $\mu: X \rightarrow \mathfrak{g}^{*}$. We suppose 
that the action extends to an action of $G^{\mathbb{C}}$, the complexifictaion of $G$. The problem is, given $x \in X$, to find $g \in G^{\mathbb{C}}$ such that $\mu(g \cdot x)=0$. Since $\mu$ is $G$-invariant, this is really a question on the symmetric space $G^{\mathbb{C}} / G$. There is a function $F: G^{\mathbb{C}} / G \rightarrow \mathbb{R}$, called the Kempf-Ness function, whose critical points correspond to solutions of $\mu(g \cdot x)=0$. Moreover, $F$ has the important property that it is convex along geodesics in $G^{\mathbb{C}} / G$. The downward gradient flow of $F$ provides a concrete way to attempt to find a zero of the moment map.

Applying this to the case of the $\mathcal{T}$-action on $\mathcal{S}^{1,1}$, we can give a moment map interpretation of some well-known facts concerning the Calabi conjecture. For a start, the symmetric space of interest is the quotient $\mathcal{T}^{\mathbb{C}} / \mathcal{T}$ of positive real functions $C^{\infty}\left(M, \mathbb{R}_{+}\right)$, or at least the open subset corresponding to $\left(\mathcal{T}^{\mathbb{C}} \cdot A\right) \cap \mathcal{S}^{1,1}$. Taking logarithms as in the discussion surrounding equations (3.2) and (3.3), we identify this space with the space of Kähler potentials

$$
\mathcal{K}=\left\{\phi \in C^{\infty}(M, \mathbb{R}): \omega_{A}+\frac{i}{2 \pi} \bar{\partial} \partial \phi>0\right\}
$$

Since $\mathcal{T}$ is an abelian group, the symmetric metric on $\mathcal{K}$ should be flat. Indeed, tangent vectors correspond to infinitesimal Kähler potentials and, given our fixed choice of volume form $\theta$, the metric is given by the $L^{2}$ innerproduct

$$
\langle f, g\rangle=\int_{X} f g \theta
$$

In particular, the geodesics for this metric are simply the affine lines in $\mathcal{K} \subset C^{\infty}(M, \mathbb{R})$.

The Kemp-Ness function is determined by the requirement that when it is pulled back to a function on $G^{\mathbb{C}}$ its derivative in the imaginary directions is given by the moment map. So, in our situation, given $A \in \mathcal{S}^{1,1}$, the derivative of the pull-back of $F$ along the path $e^{t \phi} \cdot A$ is

$$
d F(\phi)=\int_{M} \phi\left(\frac{\omega_{A}^{n}}{n !}-\theta\right) .
$$

As we saw above, on the level of Kähler forms, the tangent to the path $e^{t \phi}$ corresponds to the Kähler potential $\frac{i}{2 \pi} \bar{\partial} \partial \phi$. So we can interpret $F$ as a function on the space of Kähler potentials, given by integrating (3.4) along a path. But this is precisely the definition of a well-known energy functional, the so-called " $F_{0}$-functional", described in, for example, [Tia00]. The standard moment-map theory tells us that $F$ is convex along affine lines in $\mathcal{K}$, something which can be verified directly. As has long been observed, this fact plays an important rŹle in the study of the Calabi conjecture. In particular, since any two points of $\mathcal{K}$ lie on a geodesic, we see immediately that a solution to the Calabi conjecture must be unique. 
We can also consider the downward gradient flow of $F$. In our situation, this is the flow of Kähler metrics given by

$$
\frac{\partial \omega}{\partial t}=-\frac{i}{2 \pi} \bar{\partial} \partial\left(\frac{\omega^{n} / n !}{\theta}\right)
$$

Given Yau's solution to the Calabi conjecture, one might expect that the flow (3.5) exists for all time and converges at infinity to the solution. This has very recently been proved independently by Cao-Keller [CK11] and Fang-Lai-Ma [FLM09].

3.3. Further questions. Despite the fact that Yau has long since resolved the Calabi conjecture, this moment-map picture does raise interestings question. Typically there is a notion of "stability" associated to such a set-up; one then aims to show that a complex orbit is stable if and only if it contains a zero of the moment map. In our case, given a volume form $\theta$ we might hope to define the " $\theta$-stability" of $L \rightarrow X$. The general setup would lead us to believe that $L \rightarrow X$ is $\theta$-stable (whatever that may mean) if and only if $c_{1}(L)$ contains a solution to the Calabi conjecture. Of course, we know that this is always the case and so perhaps the sought-after definition of $\theta$-stability is something trivially satisfied by all positive bundles $L \rightarrow X$. On the other hand, Yau's solution of the Calabi conjecture is a deep result, so one might optimistically speculate that $\theta$-stability (if indeed it can be defined) is some non-trivial property of $L \rightarrow X$ implied by Yau's theorem.

There are also other versions of the Calabi conjecture which are not yet completely understood, e.g., for non-compact manifolds or singular volumeforms. To approach this problem, one might consider a modification of the setup described here, with appropriate boundary conditions at infinity or near the singularities. It would be very interesting to know if this momentmap approach sheds any light on these versions of the Calabi conjecture.

Another use of this interpretation of the Calabi conjecture may be as a testing ground for approaches to another famous - and as yet unresolved conjecture in Kähler geometry, namely the Donaldson-Tian-Yau conjecture concerning the existence of constant scalar curvature Kähler metrics (see [Don02] for a formulation of this conjecture). Since the observation of Donaldson [Don97] and Fujiki [Fuj92] that this problem can be described in terms of a moment-map, the general framework of such problems has guided much work on the subject.

With this in mind, one may attempt to reprove facts about the Calabi conjecture, directly using the moment-map formalism, and in doing so learn more about the harder problem of constant scalar curvature. While instability does not play a role in the Calabi conjecture (since a solution always exists) the comparison with constant scalar curvature metrics is certainly not 
devoid of interest. For example, just as the constant scalar curvature problem has a sequence of finite-dimensional approximations (involving Bergman spaces and balanced embeddings, see [Don01]) so does the Calabi conjecture (see [Don05]). If one could somehow use the finite-dimensional approximations to resolve problems related to the Calabi conjecture, this may shed light on exactly how to approach constant scalar curvature metrics in an analogous way.

To be more precise, we give one instance of how this might work. The flow (3.5) associated to the Calabi conjecture is known to exist for all time, but the present proofs rest on Yau's estimates. These in turn rely on the maximum principle and hence depend critically on the fact that the flow is second order. The analogous flow in the case of constant scalar curvature metrics - called the Calabi flow - is fourth order and so it is far from clear how to approach it analytically. It is for this reason that long-term existence of the Calabi flow is still an open problem.

It may be possible instead to understand the flow via a sequence of finitedimensional flows on Bergman spaces. In [Fin10] a sequence of flows on the Bergman spaces are defined and it is shown that the finite-dimensional flows converge to Calabi flow for as long as it exists. Cao and Keller [CK11] have very recently proved the analogous result in the case of the flow (3.5). Now, if one could prove directly that the finite-dimensional flows of [CK11] converge, one would have a new proof of the long-time existence of the flow (3.5) which was independent of Yau's estimates and, moreover, written in such a way as to stand a chance of generalizing to the case of the more difficult and currently rather intractable Calabi flow.

\section{References}

[AB83] M.F. Atiyah and R. Bott, The Yang-Mills equations over Riemann surfaces, Phil. Trans. R. Soc. Lond. Ser. A, 308 (1505) (1983), 523-615.

[CK11] H.D. Cao and J. Keller, About the Calabi problem: a finite dimensional approach. To appear, 2011, arXiv:1102.1097.

[Don85] S.K. Donaldson, Anti self-dual Yang-Mills connections over complex algebraic surfaces and stable vector bundles, Proc. Lond. Math. Soc. (3), 50(1) (1985), $1-26$.

[Don97] S.K. Donaldson, Remarks on gauge theory, complex geometry and 4-manifold topology, in 'Fields Medallists' Lectures, 5 of World Sci. Ser. 20th Century Math., 384-403. World Sci. Publ., River Edge, NJ, 1997.

[Don01] S.K. Donaldson, Scalar curvature and projective embeddings. I, J. Dif. Geom., 59(3) (2001), 479-522.

[Don02] S.K. Donaldson, Scalar curvature and stability of toric varieties, J. Diff. Geom., 62(2) (2002), 289-349.

[Don05] S.K. Donaldson, Some numerical results in complex differential geometry, arXiv:math/0512625v1, 2005. 
[Fin10] J. Fine, Calabi flow and projective embeddings, J. Diff. Geom., 83(3) (2010), 489-523. Appendix by K. Liu and X. Ma.

[Fin11] J. Fine, A gauge theoretic approach to the anti-self-dual Einstein equations, arXiv:1111.5005, 2011.

[FLM09] H. Fang, M. Lai and X. Ma, On a class of fully nonlinear flow in Kähler geometry, arXiv:0904.3288. To appear in Crelles J., 2009.

[Fuj92] A. Fujiki, Moduli space of polarized algebraic manifolds and Kähler metrics, [translation of Sûgaku 42 (1990), no. 3, 231-243]. Sugaku Expositions, 5(2) (1992), 173-191. Sugaku Expositions.

[GH94] P. Griffiths and J. Harris, Principles of algebraic geometry. Wiley Classics Library. John Wiley \& Sons Inc., New York, 1994. Reprint of the 1978 original.

[Kir04] A.A. Kirillov. Lectures on the orbit method, volume 64 of Graduate Studies in Mathematics. American Mathematical Society, Providence, RI, 2004.

[McD10] D. McDuff, Loops in the Hamiltonian group: a survey, in Symplectic Topology and Measure Preserving Dynamical Systems, volume 512 of Contemporary Mathematics, 127-148. Amer. Math. Soc., Providence, RI, 2010.

[Tia00] G. Tian. Canonical metrics in Kähler geometry. Lectures in Mathematics ETH Zürich. Birkhäuser, Verlag, Basel, 2000. Notes taken by Meike Akveld.

[UY86] K. Uhlenbeck and S.-T. Yau. On the existence of Hermitian-Yang-Mills connections in stable vector bundles. Commun. Pure Appl. Math., 39(S, suppl.), S257S293, 1986. Frontiers of the Mathematical Sciences: 1985 (New York, 1985).

[Wei89] A. Weinstein, Cohomology of symplectomorphism groups and critical values of Hamiltonians, Math. Z., 201(1) (1989), 75-82.

[Yau78] S.-T. Yau, On the Ricci curvature of a compact Kähler manifold and the complex Monge-Ampère equation. I, Commun. Pure Appl. Math., 31(3) (1978), 339-411.

DÉPARTMENT DE MAThÉMATIQUe,

Université LiBre de Bruxelles CP218,

Boulevard du Triomphe, Bruxelles 1050

BELGIQUE

E-mail address: joel.fine@ulb.ac.be

Received 02/03/2011, accepted 05/30/2013

It is a pleasure to acknowledge the influence of conversations with the following people: Frédéric Bourgeois, Baptiste Chantraine, Dmitri Panov, Simone Gutt, Julien Keller and Chris Woodward. I would also like to thank the referees for their insightful comments and for pointing out errors in the original manuscript. This work was written up while I was a guest at the Simons Center for Geometry and Physics, at the State University of New York, Stony Brook. I am grateful for the hospitality and the stimulating research environment which they provided. 
